\title{
Acute confusional states with right middle cerebral artery infarctions ${ }^{1}$
}

\author{
MAREK-MARSEL MESULAM, STEPHEN G. WAXMAN, NORMAN GESCHWIND, \\ AND THOMAS D. SABIN
}

From the Neurological Units, Beth Israel Hospital and Boston City Hospital; Departments of Neurology, Harvard Medical School and Tufts Medical School; and Aphasia Research Center, Department of Neurology, Boston University School of Medicine, Boston, Mass., U.S.A.

SYNOPSIS Three patients presenting predominantly with acute confusional states (ACS) are shown to have infarctions in the distribution of the right middle cerebral artery. It is suggested that the main deficit in ACS is in the function of selective attention. On the basis of cortical connections of homclogous areas in the monkey brain, it is argued that this deficit arises from lesions in convergence areas for association cortex.

We would like to report three patients whose clinical presentation was dominated by acute confusional states (ACS), and who were shown to have infarcts in the distribution of branches of the middle cerebral artery in the right cerebral hemisphere. In clinical practice, the vast majority of ACS result from metabolic encephalopathies, intoxications, withdrawal states, infections, head trauma, or post-ictal states (Cohen, 1953; Engel and Romano, 1959; Lipowski, 1967; Adams and Victor, 1974). ACS have occasionally been reported as dominating the clinical presentation of infarcts in the distribution of the posterior (Horenstein et al., 1967; Medina et al., 1974) or of the anterior (Hyland, 1933; Amyes and Nielsen, 1955) cerebral arteries. However, except for a brief description by Pearce and Miller (1973), of confusion resulting from right parietal lesions, ACS are not recognized as major sequellae of infarcts in the distribution of the middle cerebral artery. Furthermore, since the target in the central nervous system of most insults causing

1 This work was supported in part by grants NB 06209 from the National Institute of Neurological Disease and Blindness, and NS 12307 and NS-00010 from the National Institute of Health.

2 Address for correspondence: M-M Mesulam, Harvard Department of Neurology, Beth Israel Hospital, 330 Brookline Avenue, Boston, Mass. 02215, U.S.A.

(Accepted 19 August 1975.)
ACS cannot be specified, it has not been possible to localize the responsible lesion. Our purpose in this communication is, therefore, twofold first, to alert the clinician to another cerebro vascular cause of ACS; and, secondly, to advance some preliminary thoughts on the anatomy of cerebral dysfunction in ACS.

\section{CASE 1}

A 61 year old, right-handed man was admitted because of the sudden onset of agitated confusion. $\mathrm{He}$ was living alone and effectively taking care of his shopping and cooking until the day before admission when he was discovered by his landlady in a disoriented, incoherent and agitated state, banging on the doors and shouting in the middle of the night. At admission, the vital signs were unremarkable and the general physical examination was within normal limits except for a systolic ejection murmur. The neurological examination found the patient to be awake but to have a severely diminished attention span, being able to repeat only two numbers forward. Minor and irrelevant stimuli elicited dramatic orienting responses and resulted in extreme distractibility. The stream of thought was incoherent; the ability to grasp environmental cues and to react accordingly was severely compromised. There was no concern for this incapacitating change in mental status. The quantity of activity varied from sluggish- 

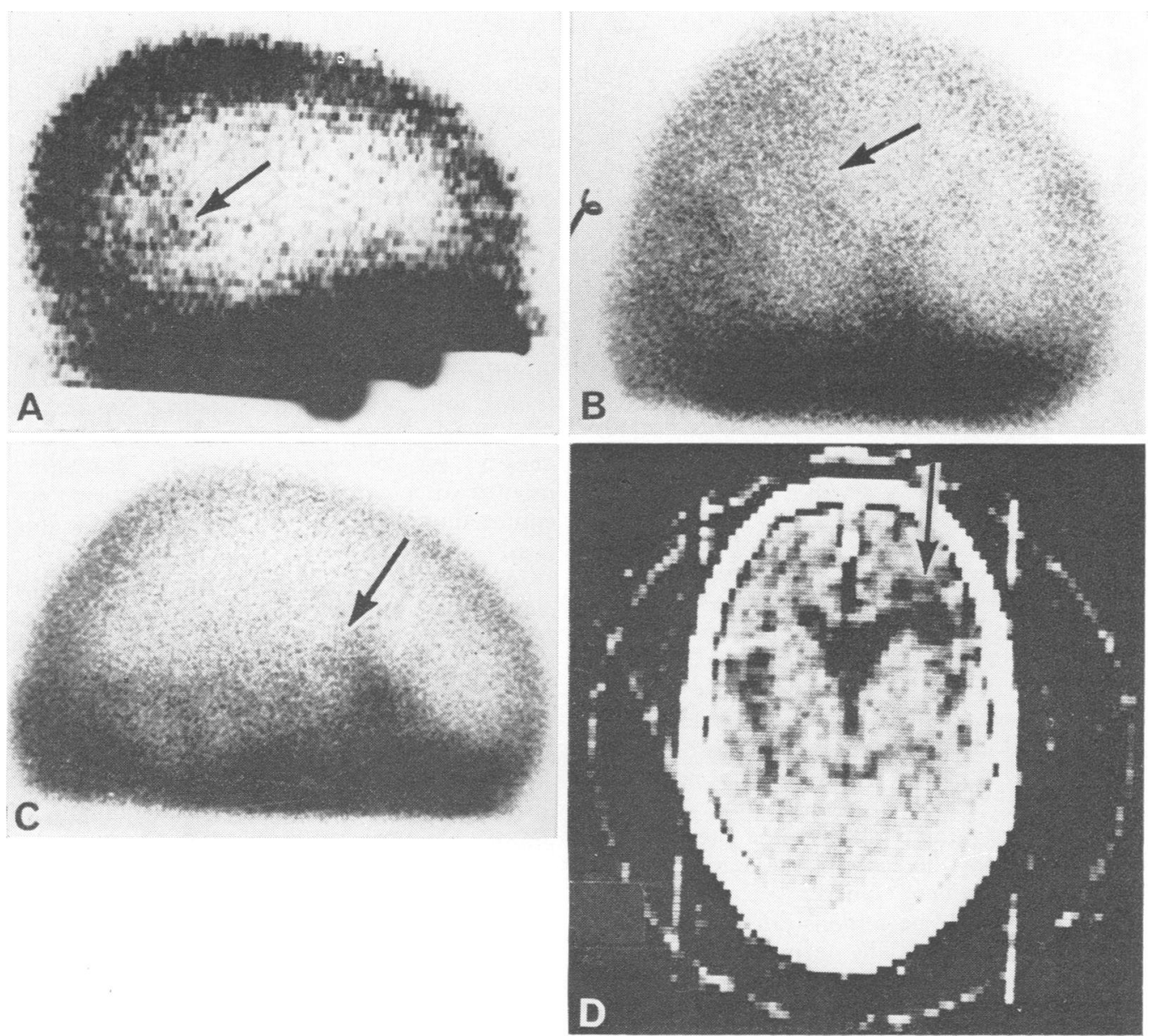

FIGURE $\mathrm{A}, \mathrm{B}$, and $\mathrm{C}$ are right lateral radioisotope scans. The anterior part of the brain is to the right. A: case 1; B: case 2; C: case 3. D is a computerized axial tomography picture of case 3. Arrows point towards the site of abnormality.

ness to agitation for which the patient required physical restraints. Several examiners thought that auditory hallucinations were present. The patient was disoriented in all spheres. He had difficulty in naming objects presented in the visual, auditory, or tactile modes and his speech contained paraphasias and circumlocutions. He could read but could not write. Extreme distractibility rendered the remainder of the mental status examination unreliable. The gait was retropulsive and unsteady. There was minimal reaction to visual stimuli in the left hemifield but the differentiation could not be made between inattention and hemianopsia. No other abnormalities of cranial nerves, sensorimotor function, or plantar responses could be detected. The patient was incontinent, unkempt, and would not use utensils for eating.

In the next few days, the agitation disappeared completely and the patient became amiable and placid. In the next month, the anomic aphasia also improved considerably; however, the attention span remained severely compromised and there was no ability to maintain a coherent stream of thought. Routine laboratory investigations were unremarkable. A technetium brain scan, which was normal at admission, revealed an area of increased uptake in the right parieto-occipital region 10 days later (Figure, A). A four-vessel arteriogram showed the 
right angular branch of the middle cerebral artery to be occluded. A pneumoencephalogram was consistent with mild cerebral atrophy.

\section{CASE 2}

A 65 year old, right-handed alcoholic man was brought to the accident floor by his landlord who reported the onset of confusion and incoherent speech four days previously. Before that time, the patient had been entirely self sufficient. The general medical examination was essentially unremarkable. The patient was fully awake but extremely inattentive and easily distractible. Neither a coherent stream of thought nor a coherent sequence of goal-directed behaviour could be maintained. He was initially agitated but this rapidly subsided into a state of placidity. Disorientation was noted in all spheres. Knowledge of remote and current world events, the ability to memorize, calculate, and abstract were all severely impaired. There was a mild naming difficulty and also dysgraphia in the absence of alexia, finger agnosia, or left-right confusion. Constructions were poorly performed. He showed severe deficits in his ability to make use of such common objects as matches or eating utensils. The gait was unsteady and retropulsive and urinary incontinence was occasionally present. The patient showed no concern for his predicament. Neglect of visual stimuli as well as minor deficits of graphaesthesia and stereognosis could intermittently be demonstrated on the left. No elementary deficits in cranial nerve or in sensorimotor functions could be demonstrated. Laboratory investigations were essentially normal. A technetium scan of the brain revealed an area of increased isotope uptake in the right parietotemporal region (Figure, B); this abnormality disappeared 16 days after admission. The patient was unchanged at discharge, one month after the onset of his illness.

\section{CASE 3}

A 74 year old right-handed male was admitted because of a sudden mental deterioration. He had been managing his personal and financial affairs successfully until the day of admission when he was discovered by his son in a disoriented and incoherent state, wearing nothing more than a necktie and a bed sheet. On admission, vital signs were unremarkable except for occasional premature ventricular contractions. The general examination showed signs of cardiomegaly and iridectomy. The mental status examination revealed minimal drowsiness, moderate inattentiveness, and agitation. The stream of thought was incoherent. His answers to questions were irrelevant and facetious. Disorientation was noted in all spheres. Details of a simple story could not be recalled five minutes later. Paraphasic errors as well as left-right confusion were noted. Cranial nerves and all sensorimotor functions, including the plantar response, were normal. There was no concern for this illness, despite the additional retropulsive gait and incontinence. Abnormal laboratory findings consisted of a blood urea nitrogen of $12.1 \mathrm{mmol} / \mathrm{l}$, blood glucose of $16.3 \mathrm{mmol} / 1$, bilirubin of $23.9 \mu \mathrm{mol} / 1$. On the third hospital day, a brain scan showed an area of increased uptake in the right inferior frontal gyrus (Figure, C, D); this area was not present on a repeat brain scan six months later. A right carotid arteriogram was negative. A computerized axial tomography of the brain localized an area in the inferior frontal gyrus of the right hemisphere with a density profile consistent with infarction. Six months after this admission, the patient was less confused but continued to display irritable sluggishness.

In summary, we have described three right-handed elderly male patients who presented with the acute onset of a confusional state. The salient and common symptoms included inattentiveness to relevant stimuli, distractibility by irrelevant stimuli, inability to grasp the immediate situation so as to react to it appropriately, inability to maintain either a coherent stream of thought or a coherent sequence of goal directed behaviour, disorientation, anomia, dysgraphia, abnormal gait, incontinence, difficulty in using common objects, and lack of concern for the illness. The level of arousal was never significantly depressed and the initial agitation rapidly resolved into a state of sluggishness. The deficits in memorizing and in intellectual functions which were also elicited may have been secondary to the inattentiveness during the testing situation. The lateralizing neurological signs were unimpressive and, indeed, were demonstrable intermittently, consisting of deficits in visual function, graphaesthesia, and stereognosis on the left. All three of these patients had radiological findings consistent with a recent right hemisphere infarct in the distribution of branches of the middle cerebral artery, two of these being situated in the general area of the inferior parietal lobule and one in the region of the inferior frontal gyrus.

\section{DISCUSSION}

CLINICAL CONSIDERATIONS The problem which brought each of these patients to the hospital consisted exclusively of behavioural abnormalities. In fact, the lateralizing neurological findings were so unimpressive that ACS secondary to toxic or metabolic encephalopathy was the 
diagnostic impression at admission in all three cases. Even when the initial agitation subsided, the left-sided deficits were demonstrable only intermittently. More detailed examination of perceptual and motor functions might well have revealed other left-sided deficits but the characteristic inattentiveness of the confused patients precluded such testing. In terms of the behavioural abnormality, moreover, the diagnosis of ACS was fully justified, since the impairments of mental status (agitation, inattentiveness, incoherent thought, cognitive deficits) and of higher cortical functions (anomia, dysgraphia, dyscalculia) is identical with that found in ACS resulting from other and more common causes (Engel and Romano, 1959; Lipowski, 1967; Chédru and Geschwind, 1972; Adams and Victor, 1974).

In addition to these cases with infarctions in the distribution of the middle cerebral artery, ACS have also been reported with occlusions of the posterior or anterior cerebral arteries (Hyland, 1933; Amyes and Nielsen, 1955; Horenstein et al., 1967; Medina et al., 1974). Our cases differ from these, since they displayed neither the persistent and extreme agitation, forced shouting, or extreme reaction to stimuli which have been noted in ACS resulting from occlusions of the posterior cerebral artery (Horenstein et al., 1967) nor the sexually inappropriate or markedly irrational behaviour described in occlusions of the anterior cerebral artery with softening in the medial frontal lobes (Hyland, 1933, case 1; Amyes and Nielsen, 1955, cases 3 and 4).

The small infarcts which our three patients suffered may be rather rare causes of ACS. In a busy neurological service, we have been able to find three definite and three other possible examples of this syndrome in a period of two years, while, during the same time, we have seen many dozens of ACS due to toxic, metabolic, traumatic, infectious, or post-ictal encephalopathies. It is conceivable that a significant number of such cases elude detection, since the paucity of elementary sensorimotor deficits in the presence of a very abnormal mental status may dissuade the primary physician from pursuing the possibility of focal neurological disease. The accurate diagnosis of these cases depends on a careful clinical assessment of focal deficits and on such laboratory investigations as the electroencephalogram, brain scan, and computerized axial tomography of the brain. Of course, each patient with an acute confusional state requires a meticulous medical assessment as well as laboratory evaluations of cerebrospinal fluid, blood, and urine, since most of these patients will suffer from a reversible toxic, metabolic, or infectious disorder which may become irreversible or fatal if not recognized and treated promptly.

ANATOMICAL CONSIDERATIONS The confusional state consists of a complex pattern of deficits in mental status. Attempts at reducing this syndrome into a disorder of one fundamental function will undoubtedly meet with objections. Nevertheless, the concept that the basic abnormality in ACS is a reduction and erratic shifting of attention (Chédru and Geschwind, 1972) is consistent with our clinical observations.

Although the definition of 'attention' is fraught with controversy (Meldman, 1970), it is generally accepted that this construct denotes at least two distinct processes. One of these is tonic and regulates the threshold which a stimulus must exceed before gaining access to consciousness; the second is phasic (selective attention) and selects, from among the many stimuli which exceed this threshold, those which will occupy the centre of awareness. The tonic process of attention is closely related to the concept of 'arousal' and, in man, this component is severely impaired with focal infarcts in the dimesencephalic junction (Segarra, 1970). In our patients, this aspect of attention was relatively well preserved. On the other hand, the proper exercise of selective attention is a far more complicated task which requires the rapid and continuous integration of environmental cues and of internal stimuli with past experiences, present needs, and expectations about the future. Furthermore, an intact capacity for selective attention would be necessary for the production of a coherent stream of thought and for the maintenance of a coherent sequence of goal-directed behaviour. It is to be expected, therefore, that selective attention is coordinated, at least in part, at a cortical level and that the cortical areas in question are association regions of the highest order where information from many other areas 
of the brain converges. It is this function of selective attention which seemed preferentially impaired in our three cases.

Although pathological examination is not available, we infer, from radiological investigations, that the lesions primarily involve the inferior frontal gyrus (case 3) and the inferior parietal lobule (cases 1 and 2). Experimental data on the connections of cortex in primates is most readily available for the rhesus monkey, in which areas 45 and 46 of Walker (1940) and the banks of the caudal superior temporal sulcus may be homologous in man to the inferior frontal gyrus and inferior parietal lobule, respectively. Analysis of corticocortical connections of these areas in the monkey brain reveals that they constitute nodal sites for the convergence of afferent fibres from secondary and tertiary association cortices in the visual, somaesthetic, and auditory modalities (Pandya and Kuypers, 1969; Pandya and Vignolo, 1969; 1970; Jones and Powell, 1970; Van Hoesen et al., 1972; Chavis and Pandya, 1974). As a possible consequence of similar convergence of neocortical afferent pathways in man, the inferior frontal gyrus (Broca's area) and the inferior parietal lobule (angular and supramarginal gyri) in the left cerebral hemisphere have crucial roles in language functions (Geschwind, 1965) but ACS do not usually result when these areas are infarcted. It is tempting to speculate, on the basis of the present cases, that the same regions in the right hemisphere are indispensable for the complex integrative processes required for the effective execution of selective attention. The notion that right hemisphere mechanisms are particularly important in maintaining selective attention is further supported by studies showing that evoked responses to visual and somatosensory stimuli have greater amplitude in the right hemisphere of normal individuals (Schenkenberg et al., 1971). There is reasonable evidence to suggest that the amplitude of such evoked responses is an index of attention (Haider et al., 1964; Sakai et al., 1966).

The confusional states associated with anterior or with posterior cerebral artery occlusions may be analysed in similar fashion since, in the monkey, the cingulate gyrus which is irrigated by the anterior cerebral artery and the inferomedial temporal cortex supplied by the posterior cerebral artery also contain convergence areas for afferents from various high order association cortices (Van Hoesen et al., 1972). Furthermore, these regions also provide direct neocortical input into such limbic structures as the amygdala (Whitlock and Nauta, 1956; Pandya et al., 1973), presubiculum (Pandya et al., 1972), and the entorhinal cortex (Van Hoesen et al., 1972; Van Hoesen and Pandya, 1975). Whereas the deficit in selective attention in these cases of ACS may result from the involvement of corticocortical convergence areas, the additional features of extreme agitation or apathy and the psychosis-like inappropriate behaviour may be conceptualized as resulting from a disconnection of limbic structures from essential neocortical input. In contrast, the agitation in our three cases with infarctions in the inferior frontal gyrus or in the inferior parietal lobule, neither of which has direct connections with the limbic brain, was mild and transient; and psychosis-like behaviour was not observed.

On the basis of these observations, several $\stackrel{\circ}{\circ}$ locations in the human nervous system may be designated where focal lesions interfere with the 8 process of attention. One such site is the dimesencephalic junction where an infarct will severelya impair 'arousal' and the related function of ? tonic attention. On the other hand, lesions in the inferior parietal lobule, inferior frontal gyrus, medial frontal lobe and inferomedial temporal lobe will cause ACS with a global deficit in selective attention. By analogy with the connections of homologous areas in the monkey brain, each one of these cortical regions may be considered as a nodal convergence site for afferent fibres from association cortex. At this point, we could not exclude the possibility that circumscribed infarctions in still other cortical sites may produce ACS. Such sites would be likely, however, to have similar corticocortical connectivity patterns for the convergence of afferent fibres from multiple association cortices in order to have an influence on the process of selective attention. In turn, the differences in clinical manifestations would be expected to reflect the unique anatomical relationships of the involved region. Furthermore, lesions in still other convergence sites in the human brain could result in unilateral trimodal neglect as suggested by Heilman and Valenstein (1972). It 
could be argued that such lesions would involve areas where the thalamic input predominates over the callosal; whereas, in the regions implicated in the aetiology of ACS the converse may be true. The dominance of thalamic influence would favour a unilateral deficit, whereas powerful callosal input would explain the global nature of the resultant symptomatology. If unilateral neglect is considered a subset of impaired selective attention, then this explanation would be consistent with the previous discussion.

These anatomical speculations must remain tentative since, in the absence of pathological examination, we cannot exclude the possibility either of antecedent lesions or of simultaneous involvement of subcortical grey matter. However, neither the past medical history of our patients nor the known territories of the implicated arterial branches support these possibilities. Furthermore, there may exist significant misgivings about the possibility of localizing, indeed of identifying, as complex a process as 'selective attention'. The heuristic value of assuming that such a unitary function exists, and that it may be localized on the basis of anatomical connectivity patterns may be evaluated only in the light of future cases.

We express our gratitude to Dr R. Duffield, Director of Nuclear Medicine at Boston City Hospital, for his gracious help in interpreting and making available the brain scans.

\section{REFERENCES}

Adams, R. D., and Victor, M. (1974). Delirium and other confusional states. In Principles of Internal Medicine, pp. 149-156. Edited by M. M. Wintrobe, G. W. Thorn, R. D. Adams, E. Braunwald, K. J. Isselbacher, and R. G. Petersdorf. McGraw-Hill: New York.

Amyes, E. W., and Nielsen, J. M. (1955). Clinicopathologic study of vascular lesions of the anterior cingulate region. Bulletin of the Los Angeles Neurological Society, 20, 112-130.

Chavis, D., and Pandya, D. N. (1974). Frontal lobe projections of the cortical sensory association areas of the rhesus monkey. Transactions of the American Neurological Association, 99, 29-32.

Chédru, F., and Geschwind, N. (1972). Disorders of higher cortical functions in acute confusional states. Cortex, 8, 395-411.

Cohen, S. (1953). The toxic psychoses and allied states. American Journal of Medicine, 15, 813-828.

Engel, G. L., and Romano, J. (1959). Delirium, A syndrome of cerebral insufficiency. Journal of Chronic Diseases, 9 , 260-277.
Geschwind, N. (1965). Disconnexion syndromes in animals and man. Part 1. Brain, 88, 237-294.

Haider, M., Spong, P., and Lindsley, D. B. (1964). Attention, vigilance, and cortical evoked potentials in humans. Science, 145, 180-182.

Heilman, K. M., and Valenstein, E. (1972). Frontal lobe neglect in man. Neurology (Minneap.), 22, 660-664.

Horenstein, S., Chamberlain, W., and Conomy, J. (1967). Infarction of the fusiform and calcarine regions: agitated delirium and hemianopia. Transactions of the American Neurological Association, 92, 85-89.

Hyland, H. H. (1933). Thrombosis of intracranial arteries. Archives of Neurology and Psychiatry (Chic.), 30, 342-356.

Jones, E. G., and Powell, T. P. S. (1970). An anatomical study of converging sensory pathways within the cerebral cortex of the monkey. Brain, 93, 793-820.

Lipowski, Z. J. (1967). Delirium, clouding of consciousness and confusion. The Journal of Nervous and Mental Disease, 145, 227-253.

Medina, J. L., Rubino, F. A., and Ross, A. (1974). Agitated delirium caused by infarction of the hippocampal formation and fusiform and lingual gyri: a case report. Neurology (Minneap.), 24, 1181-1183.

Meldman, M. J. (1970). Diseases of Attention and Perception, Pergamon: London.

Pandya, D. N., Domesick, V. B., Van Hoesen, G. W., and Mesulam, M. (1972). Projection of the cingulate gyrus and cingulum in the rhesus monkey. Anatomical Records, 172, 379.

Pandya, D. N., and Kuypers, H. G. J. M. (1969). Corticocortical connections in the rhesus monkey. Brain Research, 13, 13-36.

Pandya, D. N., Van Hoesen, G. W., and Domesick, V. B. (1973). A cingulo-amygdaloid projection in the rhesus monkey. Brain Research, 61, 369-373.

Pandya, D. N., and Vignolo, L. A. (1969). Interhemispheric projections of the parietal lobe in the rhesus monkey. Brain Research, 15, 49-65.

Pandya, D. N., and Vignolo, L. A. (1970). Intra- and interhemispheric projections of the parietal, premotor and arcuate areas in the rhesus monkey. Brain Research, 26, 217-233.

Pearce, J., and Miller, E. (1973). Clinical Aspects of Dementia, p. 41. Baillière Tindall: London.

Sakai, M., Gindy, K., and Dustman, R. (1966). Amplitude change of components of the visually evoked response as related to mental state. Proceedings of the American Psychological Association, 2, 139-140.

Schenkenberg, T., Dustman, R. E., and Beck, E. C. (1971). Changes in evoked responses related to age, hemisphere and sex. Electroencephalography and Clinical Neurophysiology, 30, 163.

Segarra, J. M. (1970). Cerebral vascular disease and behavior. Archives of Neurology, 22, 408-418.

Van Hoesen, G. W., and Pandya, D. N. (1975). Some connections of the entorhinal (area 28) and perirhinal (area 35) cortices of the rhesus monkey. 1. Temporal lobe afferents. Brain Research, 95, 1-24.

Van Hoesen, G. W., Pandya, D. N., and Butters, N. (1972). Cortical afferents to the entorhinal cortex of the rhesus monkey. Science, 175, 1471-1473.

Walker, A. E. (1940). A cytoarchitectural study of the prefrontal area of the macaque monkey. Journal of Comparative Neurology, 73, 59-86.

Whitlock, D. G., and Nauta, W. J. H. (1956). Subcortical projections from the temporal neocortex in Macaca mulatta. Journal of Comparative Neurology, 104, 183-212. 